\title{
A randomized phase II study to compare oxaliplatin plus 5-fluorouracil and leucovorin (FOLFOX4) versus oxaliplatin plus raltitrexed (TOMOX) as first-line chemotherapy for advanced colorectal cancer
}

\author{
Cristina Gravalos • Antonieta Salut • Carlos García-Girón • Rocío García-Carbonero • \\ Ana Isabel León · Isabel Sevilla • Joan Maurel • Beatriz Esteban • \\ Eduardo García-Rico • Adolfo Murias · Hernán Cortés-Funes
}

Received: 28 March 2011 / Accepted: 14 November 2011/Published online: 19 July 2012

(c) The Author(s) 2012. This article is published with open access at Springerlink.com

\begin{abstract}
Introduction The aim of this study was to compare TOMOX versus FOLFOX4 as first-line treatment of advanced colorectal cancer (CRC).

Materials and methods 191 chemotherapy-naïve patients were randomized to receive TOMOX or FOLFOX4. Patients were evaluated every 3 months and chemotherapy
\end{abstract}

C. Gravalos $(\bowtie) \cdot$ H. Cortés-Funes

Medical Oncology Department, University Hospital 12 de

Octubre, Avda. de Andalucía s/n Km 5.400,

28041 Madrid, Spain

e-mail: cgravalos@telefonica.net

A. Salut

Hospital Arnau de la Villanova, Lleida, Spain

C. García-Girón

Hospital General Yagüe, Burgos, Spain

R. García-Carbonero

Hospital Severo Ochoa, Leganés, Spain

\section{A. I. León}

Fundación Jiménez Díaz, Madrid, Spain

I. Sevilla

Hospital Virgen de la Victoria, Málaga, Spain

J. Maurel

Hospital Clinic i Provincial, Barcelona, Spain

B. Esteban

Hospital General de Segovia, Segovia, Spain

E. García-Rico

Hospital de Monteprincipe, Madrid, Spain

\section{A. Murias}

Hospital Insular de Las Palmas, Gran Canaria, Spain was continued until disease progression or unacceptable toxicity. Overall response rate was the primary endpoint. Results 183 patients were included in the intent-to-treat analysis (92 TOMOX and 91 FOLFOX4). Overall response rate was 45.6 and $36.3 \%(p=0.003)$ for TOMOX and FOLFOX4, respectively. No statistically significant differences were observed in overall survival (15.6 and 17.2 months; $p=0.475)$; progression-free survival (7.7 and 8.7 months; $p=0.292)$, and response duration (6.4 and 7.6 months; $p=0.372$ ) for TOMOX and FOLFOX4, respectively. Grades 3 and 4 neutropenia $(p<0.0001)$ and leukopenia $(p=0.028)$ were more common with the FOLFOX4 regimen, while hepatic disorders and asthenia were higher in TOMOX group ( $p=n s)$. There were two treatment-related deaths in the FOLFOX4 arm and one in the TOMOX arm. Quality of life analysis based on the SF36 revealed differences between the two regimens for physical and mental composite scores after 6 weeks, and for body pain and emotional role functioning after 6 and 12 weeks; all of these favored the FOLFOX4 arm $(p \leq 0.05)$.

Conclusions TOMOX and FOLFOX4 seem to have similar efficacy and are well tolerated in the first-line treatment for advanced CRC with different profiles of toxicity. The convenient TOMOX regimen may offer an alternative to fluoropyrimidine-based regimens.

Keywords Colorectal cancer - Raltitrexed - Oxaliplatin . Fluorouracil

\section{Introduction}

Colorectal cancer (CRC) is the second most prevalent cancer worldwide, with more than 1 million new cases 
reported per year, accounting for $9.4 \%$ of total cancers diagnosed globally [1]. In Europe, CRC is the second most commonly diagnosed cancer and accounts for $13 \%$ of newly diagnosed cases [2]. Approximately $20-30 \%$ of patients present with advanced disease. The prognosis for such patients is not promising [3], however, palliative chemotherapy increases overall survival (OS), progressionfree survival (PFS) and improved quality of life (QoL) for patients with advanced CRC, compared with best supportive care $[4,5]$.

For more than 40 years, 5-fluorouracil (5-FU)-based chemotherapy was the only treatment with activity in CRC $[6,7]$. However, during the last 10 years, a number of drugs have been demonstrated activity for advanced CRC. Oxaliplatin in combination with bimonthly 5-FU/leucovorin (LV) has been shown to be superior to 5-FU/LV in terms of response rate (RR) and PFS without having a negative impact on patients' QoL [8]. Regimens based on the De Gramont schedule combined with oxaliplatin (FOLFOX) administrated every 14 days are considered to be standard options of chemotherapy for the treatment of advanced CRC $[9,10]$, but may be unsuitable for some patients owing to the toxicity profile and inconvenient administration as it requires a central venous catheter implantation.

Raltitrexed $\left(\right.$ Tomudex $^{\circledR}$ ) is a specific inhibitor of thymidylate synthase (TS). Raltitrexed enters cells via the reduced-folate carrier and is polyglutamated by folylpolyglutamate synthase, which increases intracellular retention and leads to prolonged TS inhibition, DNA fragmentation and cell death $[11,12]$. The mechanism of action of raltitrexed differs from that of 5-FU and its serum terminal half life is longer (148-379 h) [13], which allows raltitrexed to be administered with an extended dosing interval, every 3 weeks [11].

As a single agent, raltitrexed has been extensively studied in four large comparative clinical trials that included more than 2,000 patients with advanced CRC [14-17]. In three of these studies, raltitrexed $3 \mathrm{mg} / \mathrm{m}^{2}$ every 3 weeks was compared with 5 -FU or $5-\mathrm{FU} / \mathrm{LV}$, no statistically significant differences were found in RR or survival outcomes between treatment arms [14-16]. In a fourth study, reported only as an abstract, a significantly longer median OS for 5-FU/LV was observed compared with raltitrexed at dosing of 3 or $4 \mathrm{mg} / \mathrm{m}^{2}$ [17]. Raltitrexed generally had an acceptable tolerability profile, and was associated with less leukopenia and mucositis/stomatitis than 5-FU/LV [14, 15, 17], although increased toxicity of raltitrexed compared with 5-FU/LV was reported in one study [16]. In the trial by Pazdur et al. [17] high rate of toxic death was observed when the dosing of raltitrexed was $4 \mathrm{mg} / \mathrm{m}^{2}$.
Young et al. evaluated patient preferences between raltitrexed and 5-FU-based chemotherapy regimens, regarding to adverse events and administration schedules in 82 patients with advanced CRC. Showing a similar efficacy, patients showed preferences for raltitrexed over other regimens, based its administration schedule (15-min intravenous every weeks) and/or side effects profile [18].

The extended dosing interval of raltitrexed, together with the different mechanisms of action of raltitrexed and oxaliplatin, has led to clinical interest in combining the two drugs. Raltitrexed in combination with oxaliplatin (TOMOX) has been evaluated in several preclinical and clinical studies [19-21]. Based on a phase I/II dose-escalation trial, the optimal dose of raltitrexed and oxaliplatin to be used in the TOMOX combination was established as oxaliplatin $130 \mathrm{mg} / \mathrm{m}^{2}$ combined with raltitrexed $3 \mathrm{mg} / \mathrm{m}^{2}$ every 3 weeks [19]. In phase II studies of this combination regimen, TOMOX has shown promising RR, survival and toxicity results [21-25]. These studies have provided evidence that the TOMOX combination may be effective and well tolerated in patients with advanced CRC, and have provided rationale for further evaluation of the regimen in the first-line setting.

This randomized phase II trial was designed to determine whether TOMOX is as effective as FOLFOX in the first-line treatment of advanced CRC.

\section{Materials and methods}

\section{Patients}

Patients were $\geq 18$ years with advanced, histologically or cytologically confirmed, non-resectable metastatic CRC with bi-dimensionally measurable disease, life expectancy $\geq 3$ months, Eastern Cooperative Oncology Group (ECOG) performance status $\leq 2$, adequate bone marrow (platelet count $\geq 100,000 / \mathrm{L}$, neutrophil count $\geq 2,000$ cells/L, and hemoglobin level $\geq 9.0 \mathrm{mg} / \mathrm{dL}$ ), renal [serum creatinine concentration $<1.25 \times$ upper limit of normal (ULN) and creatinine clearance $(\mathrm{CrCl})>65 \mathrm{~mL} / \mathrm{min}]$ and hepatic (serum bilirubin level $\leq 1.5 \times$ ULN, aspartate amino transferase and alanine amino transferase $\leq 2.5 \times$ ULN, and alkaline phosphatase $\leq 5 \times$ ULN) function. Exclusion criteria included previous chemotherapy for advanced disease (or adjuvant chemotherapy $\leq 6$ months before enrollment), treatment with an experimental drug within 4 weeks of inclusion, uncontrolled intercurrent disease, bone metastases as the only manifestation of the disease, any malignancy within 5 years of study entry (except for adequately treated non-melanoma skin cancer or in-situ cervical carcinoma) and grade $\geq 2$ peripheral neuropathy according to 
National Cancer Institute Common Terminology Criteria for Adverse Events (NCI-CTC) version 2.0. Pretreatment assessments included complete medical history, physical examination, performance status, complete blood count, serum chemistry, electrocardiogram, and baseline measurement of tumor size based on tomography scans (CT). The study was carried out in compliance with the Declaration of Helsinki, Good Clinical Practice Guidelines, and all applicable local regulatory requirements. Signed informed consent was obtained from all patients.

\section{Study design}

In this phase II, multicenter, open-label study, patients were randomized centrally in a $1: 1$ ratio to receive FOLFOX4 or TOMOX. Randomization was carried out using a four patient block randomization system at each study site, centrally administrated. FOLFOX4 was administered as previously described [8]: leucovorin $200 \mathrm{mg} / \mathrm{m}^{2}$, bolus 5 -FU $400 \mathrm{mg} / \mathrm{m}^{2}$ plus 22 -h continuous infusion of 5 -FU $600 \mathrm{mg} / \mathrm{m}^{2}$ days $1-2$, and oxaliplatino $85 \mathrm{mg} / \mathrm{m}^{2}$ day 1 , every 2 weeks. Patients in the TOMOX group received raltitrexed $3 \mathrm{mg} / \mathrm{m}^{2}$ as a $15-\mathrm{min}$ infusion, followed $45 \mathrm{~min}$ later by oxaliplatin $130 \mathrm{mg} / \mathrm{m}^{2}$ as a 2-h infusion, on day 1 of 3-week cycles. Raltitrexed dose and administration schedules were adjusted according to $\mathrm{CrCl}$ on day 1 of each cycle $(\mathrm{CrCl}>65 \mathrm{~mL} / \mathrm{min}: 100 \%$ of planned dose every 3 weeks; $\mathrm{CrCl}=55-65 \mathrm{~mL} / \mathrm{min}: 75 \%$ of planned dose every 4 weeks; $\mathrm{CrCl}=25-54 \mathrm{~mL} / \mathrm{min}$ : $50 \%$ of planned dose every 4 weeks). If raltitrexed administration was delayed, administration of oxaliplatin was also delayed.

Tolerability was evaluated at baseline and before each cycle. All toxicities graded according to the NCI-CTC version 2.0 except peripheral neuropathy that was evaluated according with the Sanofi classification. Dose adjustments and administration delays were evaluated based on the each patient's most severe toxicity. In FOLFOX4 group, bolus and continuous infusion 5-FU was reduced to 300 and $500 \mathrm{mg} / \mathrm{m}^{2}$, respectively, if neutropenia, thrombocytopenia, diarrhea, stomatitis, or other toxicities grade (G) 3-4 occurred. Oxaliplatin was reduced to $65 \mathrm{mg} / \mathrm{m}^{2}$ in case of paresthesias associated with pain or functional less lasted during 7-14 days, and it was stopped if longer. In the TOMOX regimen, in case of neutropenia or thrombocytopenia $\mathrm{G} 3$ or $\mathrm{G} 4$, raltitrexed was reduced to 75 or $50 \%$, respectively, and oxaliplatin was administered at $100 \mathrm{mg} / \mathrm{m}^{2}$. Regarding non-hematological toxicities, in case of diarrhea, or stomatitis G2, G3 or G4 raltitrexed was reduced at $75,50 \%$ or omitted, respectively. Oxaliplatin was reduced to $100 \mathrm{mg} / \mathrm{m}^{2}$ in case of paresthesias associated with pain or functional less lasted during 7-14 days, and was stopped if longer.
In both groups, treatment was continued until progressive disease (PD), death, withdrawal of informed consent, or unacceptable toxicity. In patients who achieved a complete response, treatment was continued for a maximum of 6 months with or without oxaliplatin, depending on degree of cumulative neurotoxicity. Prophylactic anti-emetics were administered according to normal clinical practice. Routine use of a granulocyte colony-stimulating factor was not allowed.

QoL was assessed every 6 weeks using the short form36 (SF-36) questionnaire.

The primary objective of the study was to demonstrate the non-inferiority of TOMOX when compared with FOLFOX4 in terms of Objective Response Rate (ORR). To avoid bias, response was evaluated according to RECIST every 3 months, regardless of the number of cycles administrated. The secondary objectives included OS, PFS, response duration, tolerability and QoL. OS and PFS were assessed from date of randomization until progression (PD), death or last follow-up. Response duration was defined as date of first response until $\mathrm{PD}$, death or last follow-up.

\section{Statistical analysis}

Determination of sample size was based on the primary endpoint. Based on previously published data, the ORR for both regimens was expected to be $\sim 50 \%$. The study was designed to detect a $12 \%$ as a maximum difference for non-inferiority in ORR between the two arms, using $\alpha$ of 0.05 and a $\beta$ of 0.20 . To achieve this, the number of patients required was 430 (215 per group). Intergroup comparisons of ORR $\pm 95 \%$ confidence intervals (CI) were conducted using a normal asymptotic one-sided $Z$ test for proportions (non-inferiority) on independent samples. Univariate analyses of OS and PFS were conducted according to Kaplan-Meier estimates [26]. Comparisons between survival distributions were made by Cox proportional hazards model regressions, hazard ratios (HR) and $95 \%$ CI [27]. Statistical significance was defined as $p \leq 0.05$. The limit for non-inferiority was established in $12 \%$ applied to the HR $95 \%$ CI limits.

\section{Results}

Patients

This phase II, multicenter, open-label, randomized study was conducted at 35 Spanish hospitals between January 2002 and February 2004. Due to limited funding, the study was closed prematurely for enrollment after 191 patients had been randomized. A total of 183 patients received at 


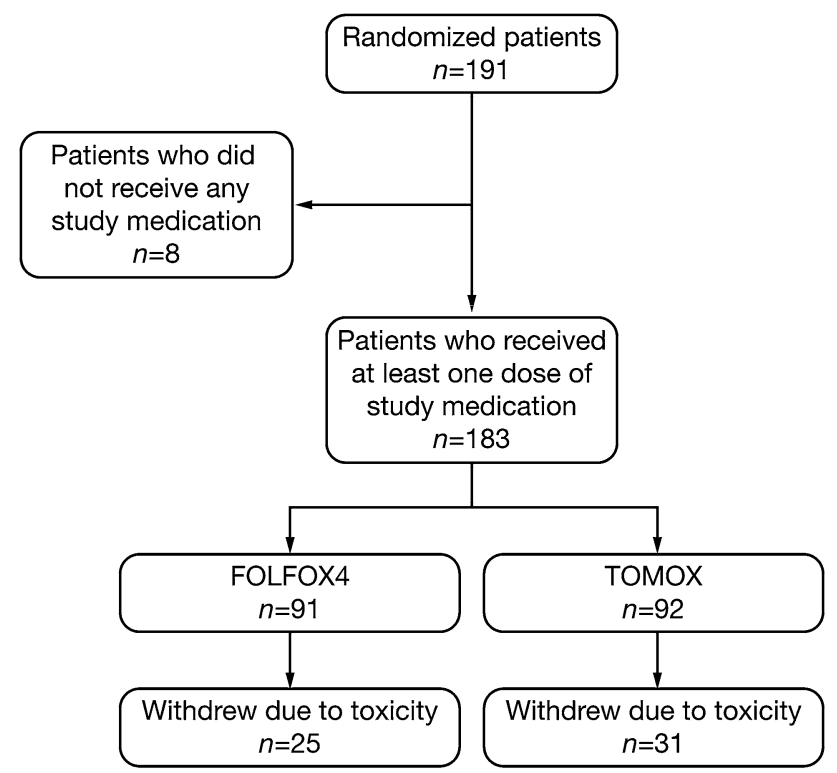

Fig. 1 Disposition of patients

least one dose of study medication and were included in the intent-to-treat population (91 in the FOLFOX4 arm and 92 in the TOMOX arm; Fig. 1). Eight patients were excluded prior to receiving study medication due to; renal function out of range ( 2 patients), hematological function out of range ( 2 patients), hepatic and renal function out of range (2 patients) and for unknown reasons (2 patients).

Baseline demographics were similar between the two treatment groups, with no significant imbalances in sex, age, ECOG score or number of organs involved (Table 1). However, there was a significant difference between the groups with respect to location of primary tumor; there was a higher prevalence of rectal tumors in the TOMOX group than in the FOLFOX4 group (43.5 vs. $24.2 \% ; p=0.005$ ).

The FOLFOX4 group received a median of eight cycles of treatment and the TOMOX group received a median of six cycles of treatment. Reasons for treatment withdrawal included toxicity (27.5 vs. $33.7 \%$ of patients, respectively), and PD (28.6 vs. $28.3 \%$ of patients, respectively). In the FOLFOX4 group, the median relative dose intensity was $84 \%$ for both 5-FU and oxaliplatin. In the TOMOX group, the median relative dose intensity was $92 \%$ for raltitrexed and $93 \%$ for oxaliplatin.

Efficacy

ORR was $36.3 \%$ for FOLFOX 4 and $45.6 \%$ for TOMOX $(p=0.0032)$ (Table 2$)$ and the non-inferiority of TOMOX in the primary endpoint when it is compared with FOLFOX4 was demonstrated. Disease control rate was similar for FOLFOX4 and TOMOX (69.3 and $74.9 \%$, respectively). With a median follow-up of 12.2 months, OS was
Table 1 Baseline characteristic of patients included in the study

\begin{tabular}{lccc}
\hline Characteristic & \multicolumn{2}{c}{ Treatment group } & \multirow{2}{*}{$p$ value } \\
\cline { 2 - 3 } & $\begin{array}{l}\text { FOLFOX4 } \\
(n=91)\end{array}$ & $\begin{array}{c}\text { TOMOX } \\
(n=92)\end{array}$ & \\
\hline Sex, $n(\%)$ & $48(52.7)$ & $56(60.9)$ & 0.2674 \\
Male & $43(47.3)$ & $36(39.1)$ & \\
Female & $61(35-82)$ & $65(36-78)$ & 0.6542 \\
Median age, years (range) & $n(\%)$ & & \\
Location of primary tumor, $n$ & $69(75.8)$ & $52(56.5)$ & 0.0058 \\
Colon & $22(24.2)$ & $40(43.5)$ & \\
Rectum & & & \\
ECOG score, $n$ (\%) & $48(52.7)$ & $51(55.4)$ & 0.5544 \\
0 & $39(42.9)$ & $39(42.4)$ & \\
1 & $4(4.4)$ & $2(2.2)$ & \\
2 & $n(\%)$ & & \\
Number of organs involved, & $1(1.1)$ & $0(0.0)$ & 0.8765 \\
0 & $59(64.8)$ & $64(69.6)$ & \\
1 & $23(25.3)$ & $20(21.7)$ & \\
2 & $8(8.8)$ & $7(7.6)$ & \\
3 & $0(0.0)$ & $1(1.1)$ & \\
4 & & & \\
\hline
\end{tabular}

ECOG Eastern Cooperative Oncology Group

Table 2 Response evaluation

\begin{tabular}{lll}
\hline Response, $n(\%)$ & \multicolumn{2}{l}{ Treatment group } \\
\cline { 2 - 3 } & FOLFOX4 $(n=91)$ & TOMOX $(n=92)$ \\
\hline Complete response & $7(7.7)$ & $4(4.3)$ \\
Partial response & $26(28.6)$ & $38(41.3)$ \\
Stable disease & $30(33.0)$ & $27(29.3)$ \\
Progressive disease & $16(17.6)$ & $12(13.1)$ \\
Not evaluable & $12(13.1)$ & $11(12.0)$ \\
\hline
\end{tabular}

$p=0.0032$ for non-inferiority of TOMOX considering overall response (complete response + partial response)

17.2 versus 15.7 months [HR 0.975 (95 \% CI 0.655, 1.451; $p=0.475$ )] (Fig. 2), PFS was 8.7 versus 7.7 months [HR 0.927 (95\% CI 0.65, 1.292; $p=0.292$ )] (Fig. 3), and response duration was 7.6 versus 6.4 months $(p=0.372)$ for FOLFOX4 and TOMOX, respectively.

Safety

All patients, who received at least one dose of study medication were evaluable for toxicity assessments. The most common grades 3-4 adverse events (AEs) are summarized in Table 3. Grades 3-4 hematologic AEs were more frequent in the FOLFOX4 group than the TOMOX group [neutropenia: 34.1 vs. $5.4 \% \quad(p<0.0001)$; 


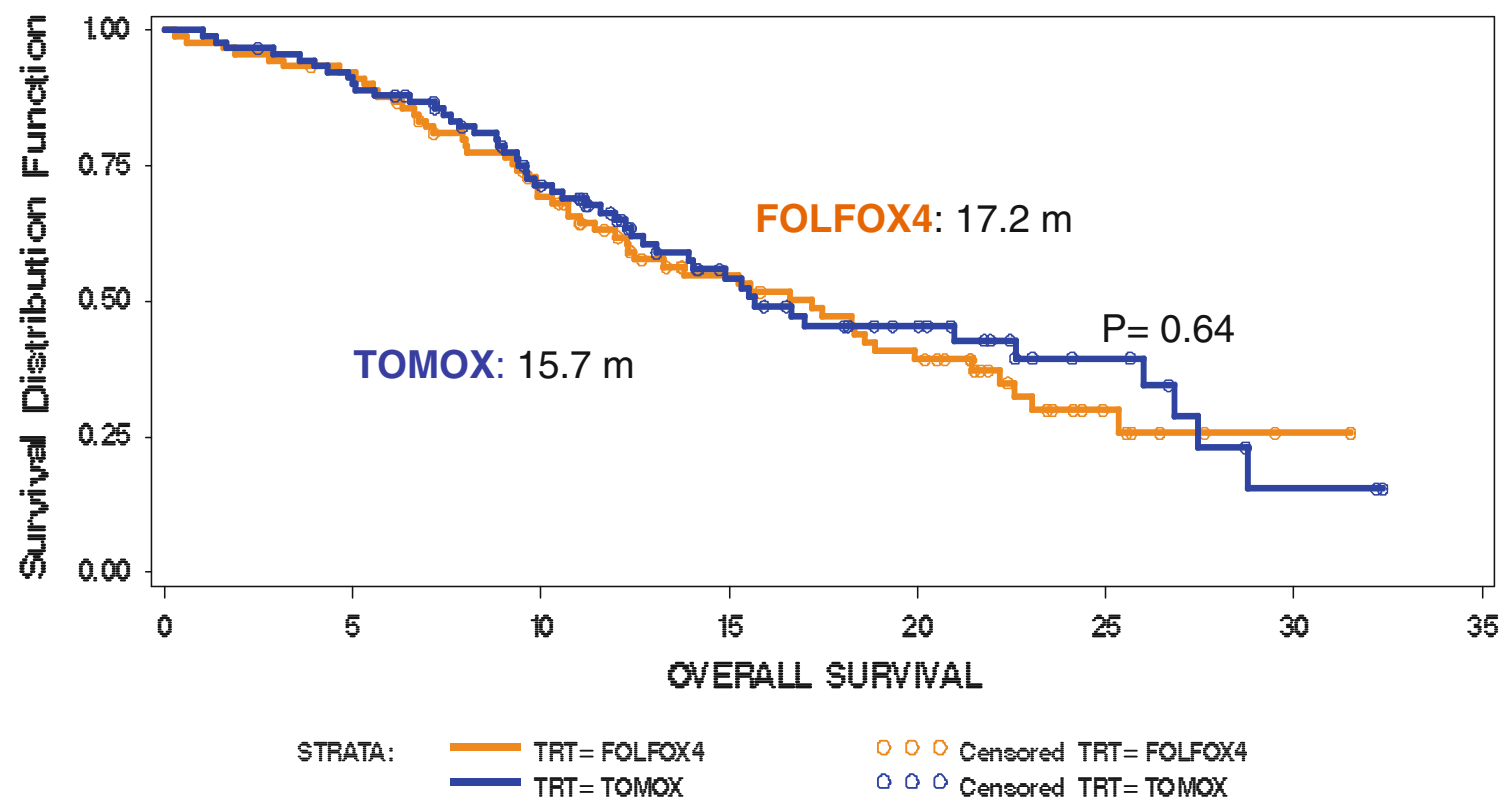

Fig. 2 Kaplan-Meier estimates of overall survival

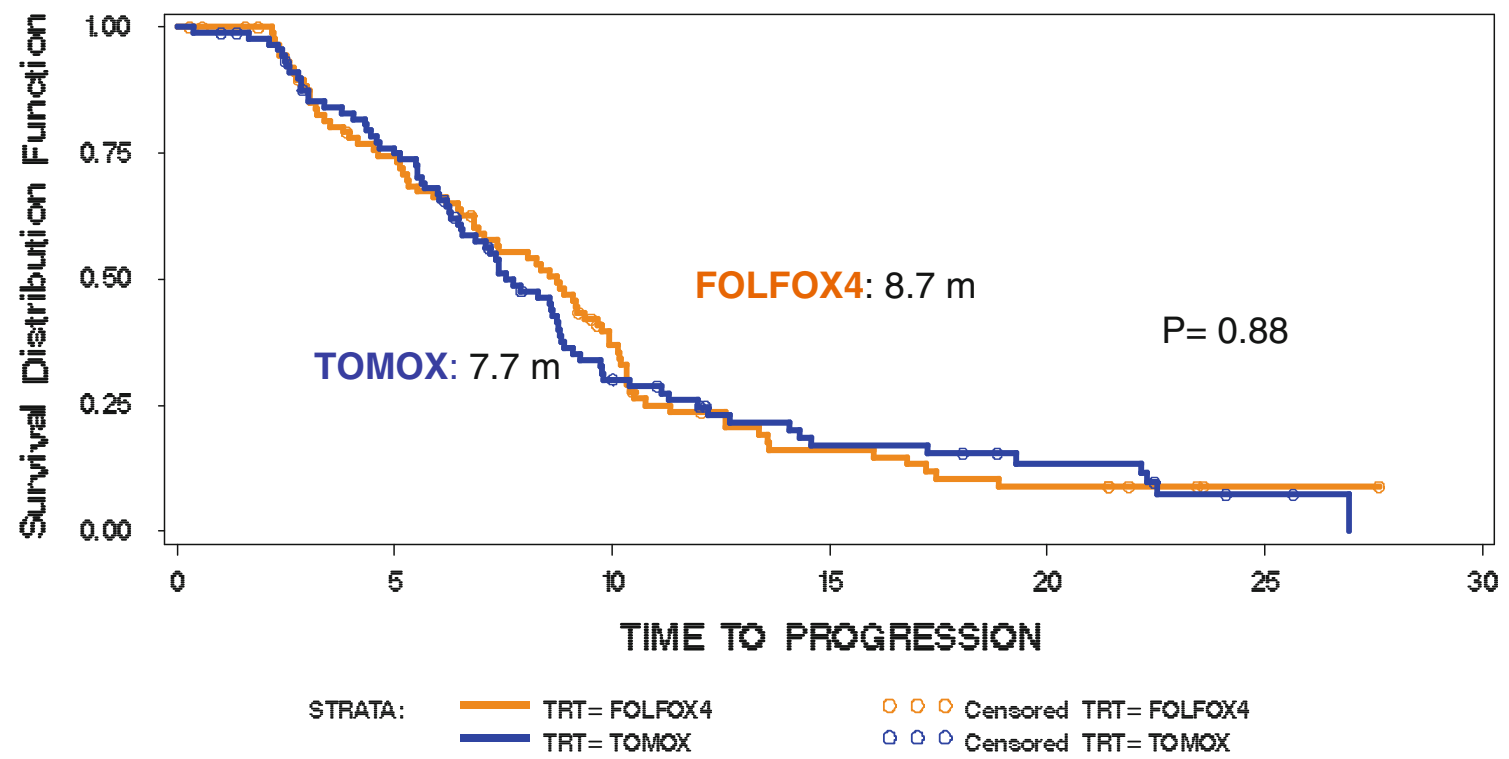

Fig. 3 Kaplan-Meier estimates of time to disease progression

leucopenia: 7.7 vs. $1.1 \%(p=0.028)$; thrombocytopenia: 6.6 vs $1.1 \%(p=0.064)]$. Hepatic disorders (25.0 vs. $14.3 \%)$ and asthenia (19.6 vs. $11.0 \%$ ) had a numerically higher incidence in the TOMOX group than the FOLFOX4 group, but the differences did not reach statistical significance. Regarding neurotoxicity, incidence of paresthesias grade $>2$ was similar for FOLFOX and TOMOX groups, (7.7 and $6.5 \%$, respectively). Sixteen patients (18.6\%) in the FOLFOX4 group and 12 patients $(13.0 \%)$ in TOMOX group experienced serious AEs (SAEs). The difference in the incidence rate of SAEs was not statistically significant $(p=0.393)$. There were two treatment-related deaths in the FOLFOX4 group (one due to neutropenic sepsis; one due to pancitopenia plus septic shock) and one in the TOMOX group (due to septic shock).

Quality of life

A total of 161 patients $(88 \%)$ were included in the QoL assessment. At baseline, there were no significant differences in scores for either composite physical/mental health measurements, or any individual components of SF-36, between the two groups. However, significant differences in SF-36 scores between the groups emerged during 
Table 3 Grades 3 and 4 hematologic and non-hematologic toxicities

\begin{tabular}{llll}
\hline $\begin{array}{l}\text { Adverse events, } \\
\%\end{array}$ patients & \multicolumn{2}{l}{ Treatment group } & \multirow{2}{l}{$p$ value } \\
\cline { 2 - 3 } & $\begin{array}{l}\text { FOLFOX4 } \\
(n=91)\end{array}$ & $\begin{array}{l}\text { TOMOX } \\
(n=92)\end{array}$ & \\
\hline Thrombocytopenia & 6.6 & 1.1 & 0.064 \\
Leukopenia & 7.7 & 1.1 & 0.028 \\
Neutropenia & 34.1 & 5.4 & $<0.0001$ \\
Diarrhea & 11 & 9.8 & NS \\
Nausea/vomiting & 11 & 6.5 & NS \\
Asthenia & 11 & 19.6 & NS \\
Hepatic disorders & 14.3 & 25 & 0.068 \\
Paresthesia & 7.7 & 6.5 & NS \\
\hline
\end{tabular}

$N S$ non-statistical significance

treatment. Both composite physical health and mental health scores were lower in the TOMOX group than the FOLFOX4 group after 6 weeks of treatment [changes in mean physical health score from baseline: +4.1 for FOLFOX4 and -2.93 for TOMOX ( $p=0.03$ ); and changes in mean mental health score from baseline: +3.7 for FOLFOX4 and -2.1 for TOMOX $(p=0.05)]$. There were no differences in composite scores after 6 weeks of treatment.

With respect to individual components of SF-36, there were significant differences in bodily pain and emotional role functioning scores in favor of FOLFOX4 after 6 and 12 weeks [bodily pain: +13.7 vs. +2.1 after 6 weeks $(p=0.05) ;+15.9$ vs. +1.85 after 12 weeks $(p=0.01)$; emotional role functioning: +13.9 vs. -4.2 after 6 weeks $(p=0.01) ;+7.1$ vs. -11.9 after 12 weeks $(p=0.05)]$.

\section{Discussion}

Over the last decade, several phase II clinical trials have assessed TOMOX in patients with metastatic CRC and have demonstrated promising results in terms of ORR, survival and tolerability [21-25, 28-30]. However, few head-to-head studies have been published that compare TOMOX with established standard of care. In this study, first-line FOLFOX4 and TOMOX showed comparable efficacy in advanced CRC with acceptable tolerability profiles. These findings suggest that TOMOX could potentially be considered as a treatment option for patients with advanced CRC.

The efficacy of TOMOX in advanced CRC observed in the current study was in accordance with previously published findings [21-25, 28-30]. The efficacy outcomes for the FOLFOX4 arm are in line with those achieved by De Gramont [8]. The median PFS reported by De Gramont et al. was 9 months, which is similar to the 8.7 months seen in our study. In contrast, the ORRs achieved in our study are lower ( 36.3 vs. $50.7 \%$ ). In the TOMOX arm of our study, the ORR, PFS and OS are within the general range obtained in previous trials. In three phase II clinical trials where patients with advanced CRC were treated with TOMOX, ORRs of $43 \%$ [24], $46 \%$ [28] and $54 \%$ [21] were reported, compared to $45.7 \%$ in our study. The median PFS values reported in the three studies $(6.2,8.2$ and 10.3 months, respectively) are also in line with the 7.7 months observed in our study. Similarly, the median OS of 15.7 months in this study is comparable to the 14.6 and 14.5 months reported for two of these studies [21, 24]. Furthermore, in a phase III clinical trial that compared TOMOX with oxaliplatin plus 5FU/LV in 216 patients [31], the TOMOX arm was superior in terms of ORR (29.1 vs. $17 \% p=0.0437$ ) although the oxaliplatin plus 5FU/ LV arm ORR was lower than previously published.

Both regimens were well tolerated, although there were some differences in the safety profiles of FOLFOX4 and TOMOX in this study. As expected, the incidence of neutropenia and leukopenia grade was higher in the FOLFOX arm and the incidence of hepatic disorders and asthenia was higher in the TOMOX arm, although without statistical significance. The number of treatment-related deaths was similar in both treatment arms ( 2 in FOLFOX arm vs. 1 in the TOMOX arm), and no unexpected AEs occurred. These data suggest that raltitrexed may be a tolerable treatment for advanced CRC, provided dose level and schedules are adjusted according to changes in $\mathrm{CrCl}$ and in response to the emergence of hematologic and nonhematologic toxicities. When compared with the data published from the PETACC1 study [32], the use of raltitrexed in our study did not increase the number of treatment-related deaths.

In contrast to 5-FU, which requires a 22-h infusion for 2 consecutive days every 2 weeks and a central venous device, raltitrexed is administered as a 15-min intravenous infusion every 3 weeks. Given that TOMOX does not seem to be associated with any additional safety and tolerability concerns compared with FOLFOX4, the relative convenience of raltitrexed administration may make TOMOX an attractive option for first-line treatment of patients with advanced CRC, particularly in patients who find it difficult to meet the scheduling commitments for FOLFOX4 infusions, who cannot tolerate 5-FU-based regimens, or who cannot have a central venous catheter [33, 34].

Our study was limited because of the sample size estimated in the statistical plan was not achieved due to funding issues. However, the overall response rate, main endpoint of the study, was not affected and the results demonstrate the non-inferiority of TOMOX treatment when it is compare with FOLFOX4. There is a lack of power to reach significance in outcomes related to survival even though all HR point estimates were very close to 1 , 
which indicates similarity between both regimens, and the non-inferiority of TOMOX could not be proven for these secondary endpoints.

Finally, it is important to note that several monoclonal antibodies have been approved for clinical use in patients with advanced CRC: the antivascular endothelial growth factor antibody bevacizumab and the anti-epidermal growth factor receptor antibodies cetuximab and panitumumab. However, targeted therapies were not standard treatment options when the current study was running. Further studies will be required to guide the optimal application of TOMOX in relation to the range of other treatment options now available for advanced CRC.

In conclusion, our study indicates that the TOMOX regimen appears similar to FOLFOX in terms of efficacy and tolerability as first-line treatment for advanced CRC. Furthermore, the ambulatory administration schedule could provide greater convenience for a large number of patients. However, confirmatory phase III studies are required to fully establish the efficacy and safety profile of the regimen. Combinations of TOMOX with monoclonal antibodies require investigation in clinical trials.

Conflict of interest The authors declare no potential conflicts of interest.

Open Access This article is distributed under the terms of the Creative Commons Attribution License which permits any use, distribution, and reproduction in any medium, provided the original author(s) and the source are credited.

\section{References}

1. International Agency for Research on Cancer (2008) World Cancer Report 2008. http://www.iarc.fr/en/publications/pdfs-online/wcr/2008/index.php

2. Boyle P, Ferlay P (2005) Cancer incidence and mortality in Europe 2004. Ann Oncol 16:481-488

3. Grothey A, Sargent D, Goldberg RM et al (2004) Survival of patients with advanced colorectal cancer improves with the availability of fluorouracil-leucovorin, irinotecan, and oxaliplatin in the course of treatment. J Clin Oncol 22(7):1209-1214

4. Chan RTT, Au GKH, Yau CC (2002) Chemotherapy for advanced colorectal carcinoma: fact and fable. Coll Radiol 5:143-154

5. Pessino A, Sobrero A (2006) Optimal treatment of metastatic colorectal cancer. Expert Rev Anticancer Ther 6(5):801-812

6. Machover D (1997) A comprehensive review of 5-fluorouracil and leucovorin in patients with metastatic colorectal carcinoma. Cancer 80(7):1179-1187

7. De Gramont A, Bosset JF, Milan C et al (1997) Randomized trial comparing monthly low-dose leucovorin and fluorouracil bolus with bimonthly high-dose leucovorin and fluorouracil bolus plus continuous infusion for advanced colorectal cancer: A French Intergroup Study. J Clin Oncol 15:808-815

8. De Gramont A, Figer A, Seymour M et al (2002) Leucovorin and fluorouracil with or without oxaliplatin as first line treatment in advanced colorectal cancer. J Clin Oncol 18:2938-2947

9. Engstrom PF, Arnoletti JP, Benson AB et al (2009) NCCN Clinical Practice Guidelines in Oncology: Colon Cancer. JNCCN 7(8):778-831
10. Engstrom PF, Arnoletti JP, Benson AB et al (2009) NCCN Clinical Practice Guidelines in Oncology: Rectal Cancer. JNCCN 7(8):838-881

11. Zalcberg JR, Cunningham D, Van Custsem E et al (1996) ZD1694: a novel thymidylate synthase inhibitor with substantial activity in the treatment of patients with advanced colorectal cancer. J Clin Oncol 14:716-721

12. Farrugia DC, Ford HE, Cunningham D et al (2003) Thymidylate synthase expression in advanced colorectal cancer predicts for response to raltitrexed. Clin Cancer Res 9:792-801

13. Beale P, Judson I, Hanwell J et al (1996) Metabolism, excretion and pharmacokinetics of a single dose of (14C)-raltitrexed in cancer patient. Cancer Chemother Pharmacol 42:71-76

14. Cunningham D, Zalcberg JR, Rath U et al (1996) Final results of a randomised trial comparing Tomudex (raltitrexed) with 5-fluorouracil plus leucovorin in advanced colorectal cancer. Ann Oncol 7:961-965

15. Cocconi G, Cunningham D, Van Cutsem E et al (1998) Open randomized multicenter trial of raltitrexed versus fluorouracil plus high-dose leucovorin in patients with advanced colorectal cancer. J Clin Oncol 16:2943-2952

16. Maughan TS, James RD, Kerr DJ et al (2002) Comparison of survival, palliation, and quality of life with three chemotherapy regimens in metastatic colorectal cancer: a multicentre randomized trial. Lancet 359:1555-1563

17. Pazdur R, Vicent M (1997) Raltitrexed (Tomudex) vs 5-fluorouracil and leucovorin $(5 \mathrm{FU}+\mathrm{LV})$ in patients with advanced colorectal cancer (ACC): results of a randomized, multicenter North American trial. Proc Am Clin Oncol 16:228 (abstract 801)

18. Young A, Topham C, Moore J et al (1999) A patient preference study comparing raltitrexed ('Tomudex') and bolus or infusional 5-fluorouracil regimens in advanced colorectal cancer: influence of side-effects and administration attributes. Eur J Cancer Care 8:154-161

19. Fizazi K, Ducreux M, Ruffie P et al (2000) Phase I dose-finding, and pharmacokinetic study of raltitrexed combined with oxaliplatin in patients with advanced cancer. J Clin Oncol 18:2293-2300

20. Fizazi K, Caliandro R, Soulié $P$ et al (2000) Combination raltitrexed (Tomu$\operatorname{dex}(\mathrm{R})$ )-oxaliplatin: a step forward in the struggle against mesothelioma? The Institut Gustave Roussy experience with chemotherapy and chemo-immunotherapy in mesothelioma. Eur J Cancer 36:1514-1521

21. Seitz JF, Bennouna J, Paillot B et al (2002) Multicenter non-randomized phase II study of raltitrexed (Tomudex) and oxaliplatin in non-pretreated metastatic colorectal cancer patients. Ann Oncol 13:1072-1079

22. Scheithauer W, Kornek GV, Ulrich-Pur H et al (2001) Oxaliplatin plus raltitrexed in patients with advanced colorectal carcinoma. Cancer 91:1264-1271

23. Scheithauer W, Kornek GV, Schuell B et al (2001) Second-line treatment with oxaliplatin + raltitrexed in patients with advanced colorectal cancer failing fluoropyrimidine/leucovorin-based chemotherapy. Ann Oncol 12:709-714

24. Neri B, Doni L, Fulignati C et al (2002) Raltitrexed plus oxaliplatin as first-line chemotherapy in metastatic colorectal carcinoma: a multicentric phase II trial. Anti Cancer Drugs 13:719-724

25. Cascinu S, Graziano F, Ferrau F et al (2002) Raltitrexed plus oxaliplatin (TOMOX) as first-line chemotherapy for metastatic colorectal cancer. A phase II study of the Italian Group for the Study of Gastrointestinal Tract Carcinomas (GISCAD). Ann Oncol 13:716-720

26. Kaplan EL, Meier P (1958) Nonparametric estimation from incomplete observation. J Am Stat Assoc 53:457-481

27. Cox DR (1972) Regression models and life tables. J R Stat Soc B 34:187-220

28. Santini D, Massacesi C, D'Angelillo RM et al (2004) Raltitrexed plus weekly oxaliplatin as first-line chemotherapy in metastatic colorectal cancer. Med Oncol 21:59-66

29. Cortinovis D, Bajetta E, Di Bartolomeo M et al (2004) Raltitrexed plus oxaliplatin in the treatment of metastatic colorectal cancer. Tumori 90(2):186-191

30. Feliu J, Castañón C, Salud A et al (2005) Phase II randomized trial of raltitrexed oxaliplatin vs raltitrexed irinotecan as first-line treatment in advanced colorectal cancer. Br J Cancer 93:1230-1235

31. Wang J, Li J, Qin S et al (2007) Randomized multicenter phase III trial of oxaliplatin plus raltitrexed compared to oxaliplatin plus fluorouracil and leucovorin treatment in recurrent and metastatic colorectal cancer. Nonparametric estimation from incomplete observation. J Clin Oncol 25:18S (Suppl). Abstract 4102

32. Popov I, Carrato A, Sobrero A et al (2008) Raltitrexed (Tomudex) versus standard leucovorin-modulated bolus 5-fluorouracil: results from the randomised phase III Pan-European Trial in Adjuvant Colon Cancer 01 (PETACC-1). Eur J Cancer 44:2204-2211

33. Volk J, Reinke F, Van Kuilenburg AB et al (2001) Safe administration of irinotecan, oxaliplatin and raltitrexed in a DPD-deficient patient with metastatic colon cancer. Ann Oncol 12:569-571

34. Luján J, García de Burgos F, Jordán A et al (2002) Angina en relación con 5-fluorouracilo. Rev Esp Cardiol 55(7):764-767 